\title{
A Rare Benign Bronchoesophageal Fistula Presenting in Adulthood: A Case Report
}

\author{
Megan Saettele ${ }^{\mathrm{a}, \mathrm{b}, \mathrm{d}}$, Nabeel Porbandarwala ${ }^{\mathrm{a}}$, David Burns ${ }^{\mathrm{c}}$
}

\begin{abstract}
We report a rare case of benign bronchoesophageal fistula (BEF) of the main left lung lower lobe bronchus associated with granulomatous disease. A30-year-old male was repeatedly seen over a period of three years at our institution for vague abdominal and respiratory symptoms. He underwent chest radiograph, CT scan of the neck, modified barium swallow study, and multiple endoscopies and laryngoscopies without a definitive diagnosis. A diagnosis of bronchoesophageal fistula was made by single contrast esophagram when a fistulous tract was demonstrated between the esophagus and left lower lobe bronchus. Chest computed tomography scanning demonstrated the fistulous tract, but also showed multiple calcified mediastinal lymph nodes adjacent to the site of fistula, which were thought to be consistent with prior granulomatous disease, likely histoplasmosis which is endemic to our area. The patient was treated surgically, and the fistula, adhesions, and adherent calcified lymph node were all removed at the time of the procedure. The presence of histoplasmosis could not be confirmed, but is most likely the cause of this uncommon BEF presentation. In conclusion, this is a rare presentation of a benign, acquired broncho-esophageal fistula associated with fungal infection.
\end{abstract}

Keywords: Bronchoesophageal fistula; Acquired fistula; Benign fistula; Histoplasmosis; Tracheoesophageal fistula; Barium esophagram

Manuscript accepted for publication February 23, 2011

${ }^{\mathrm{a}}$ University of Missouri-Kansas City, Kansas City, MO, USA

b Department of Radiology, Saint Luke’s Hospital, Kansas City, MO,

USA

${ }^{\mathrm{c}}$ National Jewish Health Center, Denver, CO, USA

${ }^{\mathrm{d}}$ Corresponding author: Megan R Saettele, Department of Radiology,

Saint Luke's Hospital and the University of Missouri-Kansas City, 4401 Wornall Road, Kansas City, MO 64111, USA.

Email: saettelem@umkc.edu

\section{Introduction}

Benign bronchoesophageal fistulas (BEF) are uncommon and are usually identified in neonates. Adult bronchoesophageal fistulas are typically of acquired malignant, traumatic, or infectious origin. Here we report the case of a thirty-yearold male who presented with a variety of respiratory and abdominal symptoms over a period of three years in which the diagnosis of a bronchoesophageal fistula was made with a modified barium swallow and etiology suggested by subsequent CT.

\section{Case Report}

A 30-year-old male presented to the otolaryngology clinic

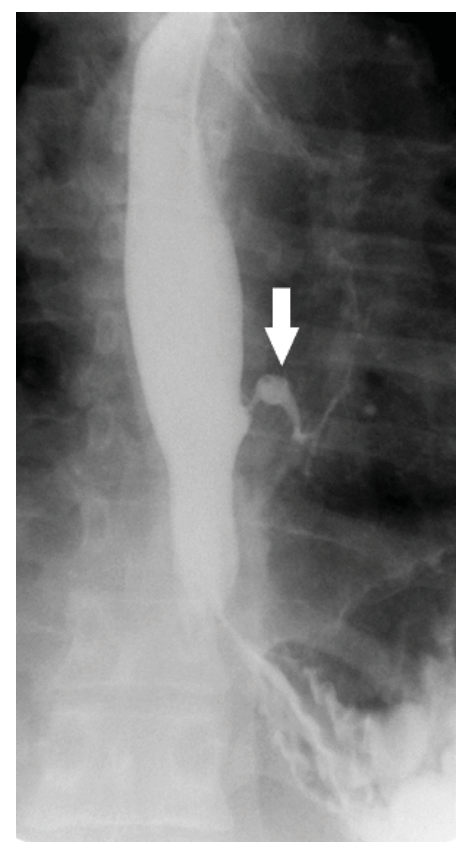

Figure 1. Spot fluoroscopic image from a single contrast esophagram demonstrates a fistulous tract between the lateral esophageal wall and the left lower lobe bronchus. 


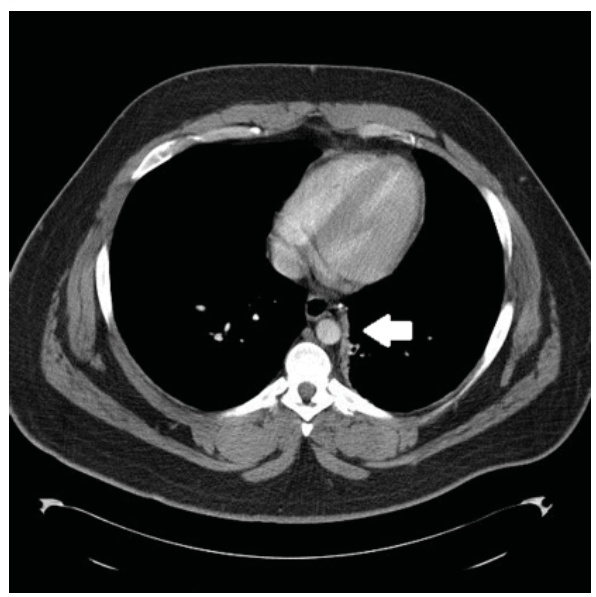

Figure 2. Axial contrast enhanced chest CT image shows the fistulous tract between the esophagus and left lower lobe bronchus.

with chief complaint of coughing while eating and drinking followed by occasional emesis, as well as coughing that awakened him at night. These symptoms had persisted for two to three years before presentation. His past medical history was significant for gastroesophageal reflux disease, asthma, post-nasal drip, alcohol use, and two recent upper respiratory infections. The patient had previously undergone a chest $\mathrm{x}$-ray, swallow study, modified barium swallow, CT scan of the soft tissues of the neck, three upper endoscopies, and two fiber optic laryngoscopies in the previous three years, all of which were negative as to the cause of his symptoms. A single contrast esophagram was performed subsequently and demonstrated a tract between the distal lateral esophagus connecting with the main left lower lobe bronchus (Fig. 1). Chest CT with contrast showed a fistulous tract between the esophagus and the main left lower lobe bronchus approximately $5 \mathrm{~cm}$ above the gastroesophageal junction with an adjacent calcified granuloma as well as multiple other calcified granulomas throughout the mediastinum (Fig. 2).

The patient elected to undergo left thoracotomy with decortication to remove the broncho-esophageal fistula. At the time of surgery, a bronchoscopy was performed that showed frothy secretions originating from the left lower lobe, but no endobronchial lesions were noted. Endoscopy was also performed which demonstrated a fistula at $38 \mathrm{~cm}$ from the incisors, which consisted of a 5 - $6 \mathrm{~mm}$ diameter epithelialized, musculous tract into the lung (Fig. 3). During thoracotomy, adhesions to the lower lobe and a calcified granulomatous nodule were identified in the area adjacent to the fistula and excised. An intercostal muscle flap interposition was performed with Heller myotomy and Belsey Mark IV fundoplication before closing. Pathology confirmed an epithelialized tract with a muscular layer, as well as chronic granulomatous inflammation, however no pathogen was identified. A repeat post-operative esophagram showed no evidence of broncho-

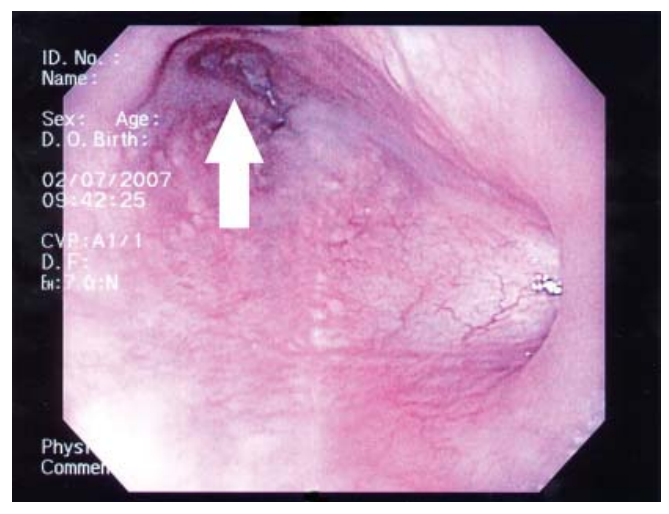

Figure 3. Endoscopic image shows fistulous opening along the lateral esophageal wall.

esophageal fistula or esophageal leak. The patient made a full recovery with no complications or recurrence of symptoms.

\section{Discussion}

Benign bronchoesophageal fistulas are rare in adults. They can be either congenital or acquired. Congenital BEFs are usually diagnosed in neonates because they are frequently associated with bronchopulmonary and/or foregut malformations such as esophageal atresia, whereas acquired BEFs can arise at any point in one's lifetime [1]. Acquired BEFs are more common and are due to malignant disease of the chest approximately $80 \%$ of the time. Cases of acquired BEFs due to chronic infections such as tuberculosis, histoplasmosis, actinomycosis, and syphilis have been reported, as well as injuries due to radiation, post-surgical or postendoscopic lesions, local trauma due to burns or penetration, and broncholithiasis [2, 3].

Bronchoesophageal fistulas commonly communicate with the right lung, and fewer cases have been reported of left lung BEFs. This has been hypothesized to be due to a thinner muscle layer and lack of soft tissue envelope around the esophagus below the carina, causing a predilection for fistula formation in the right lung lobes [4]. We do not know whether our patient's BEF was caused by histoplasmosis or not, because pathology was unable to find a pathogen, but CT findings and the endemic location of the patient make it a high possibility. The pathophysiology of BEFs due to granulomatous disease has been reported by Wychulis et al (1966) and Gerzic et al (1990), and begins with inflamed lymph nodes that become adherent to adjacent esophageal wall. Healing of those nodes produces scarring and contracture, which pulls on the esophageal wall creating a traction diverticulum that commonly is asymptomatic. With time, the enlarged and/or calcified lymph nodes adhere to the diverticulum and an airway, and when enough mediastinal 
movement or local erosion is present, a BEF can develop $[5,6]$.

Symptoms that should raise the suspicion of a bronchoesophageal fistula in an adult include: recurrent bronchopulmonary infections, dysphagia, asthma-like crises, coughing on liquid ingestion, vague thoracic or epigastric pain, hemoptysis, hematemisis, heartburn, and weight loss. Diagnosis of BEFs can be challenging. There are many diagnostic tools to help with visualization of a BEF, but as was seen in our patient, sometimes achieving a diagnosis requires time and multiple modalities; however, single contrast barium esophagography remains the modality of choice due to its sensitivity and ability to make the diagnosis.

Immediate surgical treatment is recommended for a finding of BEF despite its seemingly benign nature. Surgical treatment has the most favorable prognosis, but endoscopic occlusion with silver nitrate, biological glue, or a Celestin tube is also an option if a patient cannot tolerate a surgical procedure [7].

Confirmation of a BEF as congenital or acquired in origin may be established by Brunner's criteria (1961) [8]. Criteria for a congenital BEF include the absence of past or present surrounding inflammation, absence of adherent lymph nodes, and the presence of an epithelium-lined tract with a muscular layer. Although our patient was found to have a completely epithelialized tract with a muscular layer, criteria for congenital origin cannot be fulfilled due to inflammation surrounding the fistula, as well as an adherent calcified lymph node. Despite a negative pathogen staining, and absence of testing for fungal antigens, histoplasmosis is the probable cause of our patient's acquired BEF.

\section{Conflicts of Interest}

None

\section{Abbreviations}

BEF: bronchoesophageal fistula (A fistula connecting the esophagus to a bronchus or other lung structure)

\section{References}

1. Aguilo R, Minguella J, Jimeno J, Puig S, Galeras JA, Gayete A, Sanchez-Ortega JM. Congenital bronchoesophageal fistula in an adult woman. J Thorac Cardiovasc Surg 2006;131(4):916-917.

2. Deb S, Ali MB, Fonseca P. Congenital bronchoesophageal fistula in an adult. Chest 1998;114(6):1784-1786.

3. Kaul DR, Orringer MB, Saint S, Jones SR. Clinical problem-solving. The Drenched Doctor - a 55-yearold male physician was seen in August because of a 1-week history of fever and night sweats. N Engl J Med 2007;356(18):1871-1876.

4. Shimada T, Abo S, Kitamura M, Hashimoto M, Shikama T, Kimura Y. [A case of congenital esophago-bronchial fistula communicated between esophageal diverticulum and left main bronchus in the adult--a review of 47 cases in the Japanese literature]. Nippon Kyobu Geka Gakkai Zasshi 1992;40(11):2100-2106.

5. Wychulis AR, Ellis FH, Jr., Andersen HA. Acquired nonmalignant esophagotracheobronchial fistula. Report of 36 cases. JAMA 1966;196(2):117-122.

6. Gerzic Z, Rakic S, Randjelovic T. Acquired benign esophagorespiratory fistula: report of 16 consecutive cases. Ann Thorac Surg 1990;50(5):724-727.

7. Cossentino MJ, Ormseth EJ, Tavaf-Motamen H, Cheney CP. Congenital bronchoesophageal fistula in the adult: a case report. Am J Gastroenterol 2000;95(8):2116-2118.

8. Brunner A. [Esophagobronchial fistulae]. Munch Med Wochenschr 1961;103:2181-218 